\title{
Ultrastrong-coupling phenomena beyond the Dicke model
}

\author{
Tuomas Jaako, ${ }^{1, *}$ Ze-Liang Xiang, ${ }^{1}$ Juan José Garcia-Ripoll, ${ }^{2}$ and Peter Rabl ${ }^{1}$ \\ ${ }^{1}$ Vienna Center for Quantum Science and Technology, Atominstitut, TU Wien, Stadionallee 2, 1020 Vienna, Austria \\ ${ }^{2}$ Instituto de Física Fundamental, IFF-CSIC, Calle Serrano 113b, E-28006 Madrid, Spain
}

(Received 3 August 2016; published 27 September 2016)

\begin{abstract}
We study effective light-matter interactions in a circuit QED system consisting of a single $L C$ resonator, which is coupled symmetrically to multiple superconducting qubits. Starting from a minimal circuit model, we demonstrate that, in addition to the usual collective qubit-photon coupling, the resulting Hamiltonian contains direct qubit-qubit interactions, which have a drastic effect on the ground- and excited-state properties of such circuits in the ultrastrong-coupling regime. In contrast to the superradiant phase transition expected from the standard Dicke model, we find an opposite mechanism, which at very strong interactions completely decouples the photon mode and projects the qubits into a highly entangled ground state. These findings resolve previous controversies over the existence of superradiant phases in circuit QED, but they more generally show that the physics of two- or multiatom cavity QED settings can differ significantly from what is commonly assumed.
\end{abstract}

DOI: 10.1103/PhysRevA.94.033850

\section{INTRODUCTION}

The Dicke model (DM) is frequently used in atomic and solid-state systems as a minimal model to describe phenomena related to the collective coupling of many emitters to a single radiation mode [1,2]. When extended to the ultrastrong-coupling regime, where the collective coupling becomes comparable to the atomic and optical frequencies, the ground state of the DM undergoes a phase transition into a superradiant state [3-5]. This state is characterized by a nonvanishing field expectation value and a uniform atomic polarization and is commonly considered the hallmark of ultrastrong-coupling physics. While this superradiant phase transition (SRT) is well understood and has been observed with engineered Hamiltonians in driven atomic systems [6-8], the validity of the Dicke model for describing also the ground states of equilibrium cavity QED systems is still the subject of debate [9-14]. This question has regained considerable interest with the development of circuit QED systems [15-18], where superconducting two-level systems are strongly coupled to microwave photons. In particular, it has been argued [19] that the equivalent of the so-called $A^{2}$ term-which supposedly prevents the SRT in atomic systems [9] - does not play a crucial role in these artificial circuit QED devices. However, these predictions have also been questioned on very general grounds [20] or based on concrete models [21]. While recently the ultrastrong-coupling regime for single qubits has been experimentally achieved [22-26], the nature of the ground states of collective circuit QED systems remains open.

In this work we investigate a multiqubit generalization of a circuit QED system, where $N$ charge qubits are coupled symmetrically to a single microwave mode. In the limit of weak coupling the system reduces to the standard DM and thus such circuits have been proposed for studying the superradiant phase transition $[19,27,28]$, which is expected to occur when either the coupling strength or the number of qubits is increased. The important finding of this work is that the full Hamiltonian for this circuit necessarily contains

\footnotetext{
*tuomas.jaako@ati.ac.at
}

additional direct qubit-qubit interactions, which have been ignored in many previous studies but become nonnegligible as one approaches the ultrastrong-coupling regime. The analysis of the ground-state properties of this extended Dicke model (EDM) reveals surprising new effects, which completely contradict our existing intuition about light-matter interaction. Instead of undergoing a transition into a superradiant phase with increasing coupling strength, the system first gradually evolves into a hybridized qubit-photon state, but without broken symmetry. At even higher interaction strengths, an opposite effect can take place, where the photonic component of the ground state completely decouples, while the qubits collapse into a highly entangled Dicke state with vanishing dipole moment. We explain these findings with an effective low-energy theory, which reveals the existence of separate manifolds with an exponentially large number of nearly degenerate states. Together with the high degree of entanglement, this feature makes the EDM considerably more involved than the well-studied DM and imposes many new challenges for theoretical and experimental research on ultrastrong-coupling physics.

The analysis presented in this work is primarily focused on a minimal model for a charge-coupled circuit, which is motivated by previous studies on this subject and allows a simple physical interpretation of the predicted effects. It also establishes a direct connection to the single-mode Hopfield model that is used to describe ultrastrong-coupling effects in other electrically coupled cavity QED settings [29-31], or related models for coupled quantum dots [32], and shows the broader scope of our findings. However, the EDM can also be derived for inductively coupled circuits (although not for all [33]) based on the flux qubit design. For this type of a qubit, the ultrastrong-coupling regime is already experimentally accessible today [25,26], and using the tunability of the coupling $[25,26,34]$ in these setups, it should be possible to dynamically test the predicted new effects for $N=2$ or more qubits, over a wide range of coupling strengths, including the scaling of the qubit-qubit interaction strength, light-matter decoupling, and ground-state properties.

The remainder of the paper is structured as follows. In Sec. II we derive the exact Hamiltonian for a minimal collective 

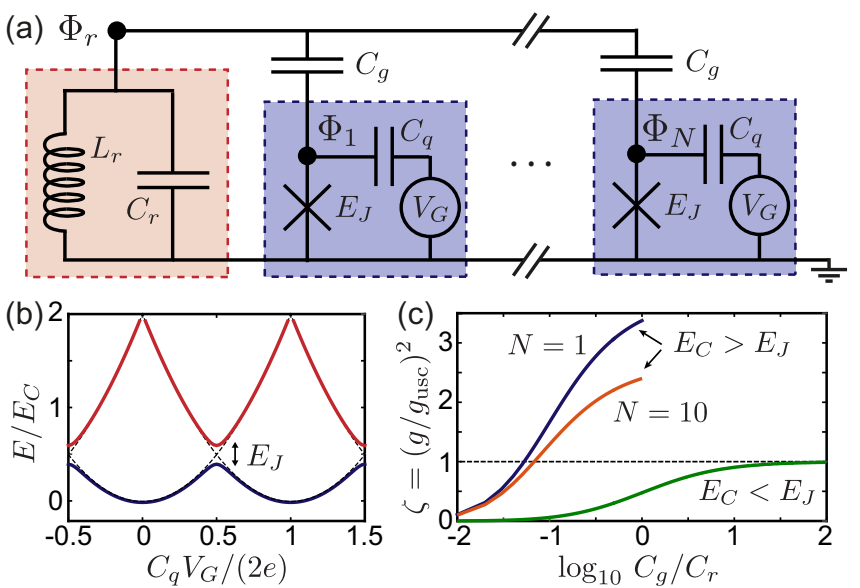

FIG. 1. (a) Circuit model for $N$ charge qubits coupled to a single lumped-element resonator. (b) Sketch of the energy levels of a single charge qubit as a function of the applied gate voltage and for $E_{C} \gg$ $E_{J}$. (c) Plot of the coupling parameter $\zeta=\left(\mathrm{g} / g_{\text {usc }}\right)^{2}$ as a function of $C_{g} / C_{r}$ and a specific set of circuit parameters $C_{r}=10 \mathrm{fF}, C_{q}=$ $0.5 \mathrm{fF}$, and $L_{r}=1 \mathrm{nH}$. For the upper two lines $E_{J} / h=2 \mathrm{GHz}$ and the condition $E_{J} / E_{C}<1$ is fulfilled over the whole range of plotted values. The lower line represents the transmon limit $\left(E_{J} / E_{C}>1\right)$ given in Eq. (7) for $N=1$.

circuit QED systems and in Sec. III we discuss the meaning of superradiant states, first for the example of a single qubit. In Sec. IV we then analyze the ground-state properties of the collective model and explain the origin of the light-matter decoupling effect. In Sec. V we briefly describe alternative circuit implementations based on the flux qubit design and demonstrate the robustness of the observed effects with respect to experimental imperfections. Finally, Sec. VI summarizes the main conclusions of this work.

\section{COLLECTIVE CIRCUIT QED}

We consider a superconducting circuit as depicted in Fig. 1(a), where $N$ charge qubits $[18,35]$ are coupled symmetrically to a single-mode lumped element resonator with capacitance $C_{r}$ and inductance $L_{r}$. Each qubit is represented by a Cooper pair box with capacitance $C_{q}$ and Josephson energy $E_{J}$ and coupled to the $L C$ resonator via an additional capacitance $C_{g}$. The whole circuit is described by the Lagrangian

$$
\mathcal{L}=\frac{C_{r} \dot{\Phi}_{r}^{2}}{2}-\frac{\Phi_{r}^{2}}{2 L_{r}}+\sum_{i=1}^{N}\left[\mathcal{L}_{q}\left(\Phi_{i}, \dot{\Phi}_{i}\right)+\frac{C_{g}}{2}\left(\dot{\Phi}_{r}-\dot{\Phi}_{i}\right)^{2}\right]
$$

where $\Phi_{\eta}(t)=\int_{-\infty}^{t} V_{\eta}(s) d s$ is the generalized flux [36] associated with the voltage $V_{\eta}$ at each node, $\eta=r, 1, \ldots, N$ [see Fig. 1(a)]. The Lagrangian for each qubit is

$$
\mathcal{L}_{q}(\Phi, \dot{\Phi})=\frac{C_{q}}{2}\left(\dot{\Phi}-V_{G}\right)^{2}+E_{J} \cos \left(\frac{\Phi}{\Phi_{0}}\right),
$$

where $V_{G}$ is the applied gate voltage and $\Phi_{0}=\hbar /(2 e)$ is the reduced flux quantum. We emphasize that apart from the coupling of each individual qubit to the cavity, our model in Eq. (1) does not contain any direct capacitive interactions among the qubits themselves.

To obtain a quantized circuit model, we follow the standard quantization procedure and replace the $\Phi_{\eta}$ and the conjugate node charges $Q_{\eta}=\partial \mathcal{L} / \partial \dot{\Phi}_{\eta}$ with operators obeying $\left[\Phi_{\eta}, Q_{\eta^{\prime}}\right]=i \hbar \delta_{\eta \eta^{\prime}}$. For the resulting Hamilton operator $H=$ $\sum_{\eta} Q_{\eta} V_{\eta}-\mathcal{L}$ we then obtain (see Appendix A)

$$
H=\frac{Q_{r}^{2}}{2 \bar{C}_{r}}+\frac{\Phi_{r}^{2}}{2 L_{r}}+\sum_{i=1}^{N}\left[H_{q}^{i}+\frac{Q_{r} \mathcal{Q}_{i}}{\bar{C}_{g}}\right]+\sum_{i \neq j} \frac{\mathcal{Q}_{i} \mathcal{Q}_{j}}{2 \bar{C}_{q q}}
$$

where $\mathcal{Q}_{i}=Q_{i}+C_{q} V_{G}$ is the displaced charge and

$$
H_{q}^{i}=\frac{\mathcal{Q}_{i}^{2}}{2 \bar{C}_{q}}-E_{J} \cos \left(\frac{\Phi}{\Phi_{0}}\right)
$$

is the Hamiltonian for an individual qubit. The modified capacitances that appear in Eqs. (3) and (4) are given by $\bar{C}_{q}=\bar{C}^{2} /\left[C_{r}+C_{g}+(N-1) C_{g} C_{q} /\left(C_{q}+C_{g}\right)\right], \quad \bar{C}_{r}=$ $\bar{C}^{2} /\left(C_{q}+C_{g}\right), \bar{C}_{g}=\bar{C}^{2} / C_{g}, \bar{C}_{q q}=\left(C_{g}+C_{q}\right) \bar{C}^{2} / C_{g}^{2}$, and $\bar{C}^{2}=C_{g} C_{r}+C_{q}\left(C_{r}+N C_{g}\right)$.

Equation (3) first shows that the coupling of individual qubits to a common resonator mode renormalizes the bare resonator and qubit energies. This is analogous to the effect of the $A^{2}$ term, which in atomic cavity QED systems increases the photonic field energy proportional to the number of atoms and can thereby prevent an SRT [9]. Interestingly, here the coupling lowers the charging energies, i.e., $\bar{C}_{r, q}>C_{r, q}$, which is exactly the opposite effect and would, at first glance, favor a SRT. However, from the last term in Eq. (3) we also see that the Legendre transformation from voltage to charge variables introduces additional direct qubit-qubit interactions, the effect of which we analyze in the following.

In the final step we express $Q_{r}=Q_{0}^{r}\left(a^{\dagger}+a\right)$ and $\Phi_{r}=$ $i Q_{0}^{r} /\left(\bar{C}_{r} \bar{\omega}_{r}\right)\left(a^{\dagger}-a\right)$ in terms of annihilation and creation operators, $a$ and $a^{\dagger}$, where $\bar{\omega}_{r}=1 / \sqrt{L_{r} \bar{C}_{r}}$ and $Q_{0}^{r}=$ $\sqrt{\hbar \bar{C}_{r} \bar{\omega}_{r} / 2}$. We further restrict each qubit to the two lowest states $|0\rangle$ and $|1\rangle$ (at this stage, without further justification), which are separated by an energy $\hbar \bar{\omega}_{q}$. For the examples considered below we can then write $H_{q}^{i} \simeq \hbar \bar{\omega}_{q} \sigma_{z}^{i} / 2$ and $\mathcal{Q}_{i} \simeq Q_{0}^{q} \sigma_{x}^{i}$, where $Q_{0}^{q}=\langle 1|\mathcal{Q}| 0\rangle$ and the $\sigma_{k}$ are the usual Pauli operators. Under these assumptions and by introducing collective spin operators $S_{k}=\frac{1}{2} \sum_{i} \sigma_{k}^{i}$, we finally obtain $(\hbar=1)$

$$
H \simeq \bar{\omega}_{r} a^{\dagger} a+\bar{\omega}_{q} S_{z}+g\left(a+a^{\dagger}\right) S_{x}+D S_{x}^{2},
$$

where $g=2 Q_{0}^{r} Q_{0}^{q} /\left(\hbar \bar{C}_{g}\right)$ and $D=2\left(Q_{0}^{q}\right)^{2} /\left(\hbar \bar{C}_{q q}\right)$. Equation (5) thus represents the minimal model for $N \geqslant 2$ qubits that are coupled capacitively to a single microwave mode. Below we show that identical models can be derived for basic flux-coupled circuits, and these models are also discussed in other cavity QED implementations [30,31]. In the absence of the last term, Eq. (5) reduces to the standard DM, which exhibits a superradiant ground state for couplings $\sqrt{N} g \geqslant$ $g_{\text {usc }}$ [5], where we use $g_{\text {usc }}=\sqrt{\bar{\omega}_{r} \bar{\omega}_{q}}$ to define the onset of the ultrastrong-coupling regime for a single qubit. However, since they have the same physical origin the qubit-qubit interaction strength $D$ and the qubit-resonator coupling $g$ are not independent. In view of $\bar{C}_{q q}=\bar{C}_{g}^{2} / \bar{C}_{r}$ in Eq. (3), we obtain 
the exact relation

$$
D=\frac{g^{2}}{\bar{\omega}_{r}},
$$

which demonstrates the significance of this additional term in the limit of very strong interactions.

\section{ULTRASTRONG COUPLING}

Before we proceed with the multiqubit model let us first evaluate the value of the single-qubit coupling parameter $\zeta=\left(g / g_{\text {usc }}\right)^{2}$ that can actually be achieved for a specific circuit design. We first do so for the frequently used transmon qubit [37], which is operated in the regime $E_{J} \gg E_{C}=$ $e^{2} /\left(2 \bar{C}_{q}\right)$ and $V_{G}=0$. In this case the two lowest eigenstates are well approximated by harmonic oscillator states with $\bar{\omega}_{q} \simeq \sqrt{8 E_{C} E_{J}}$ and $Q_{0}^{q} \simeq \sqrt{\hbar \bar{C}_{q} \bar{\omega}_{q} / 2}$ and we obtain

$$
\zeta=\frac{C_{g}^{2}}{C_{r}\left(C_{g}+C_{q}\right)+C_{g}\left(C_{g}+N C_{q}\right)}<1 .
$$

This shows that, independently of the circuit parameters, the single-qubit ultrastrong-coupling regime cannot be reached. Such a limit on the coupling parameter is consistent with more general no-go theorems discussed in Ref. [20] or the absence of an SRT found in other explicit multiqubit circuit QED models [21]. It can be traced back to the fact that in harmonic or weakly nonlinear systems the coupling $g \sim \sqrt{\bar{\omega}_{q} \bar{\omega}_{r}}$ is directly related to the qubit and the resonator frequency. To break this relation we now consider instead the charge qubit limit $E_{J} \ll E_{C}$ and $C_{q} V_{G} /(2 e)=1 / 2$ [see Fig. 1(b)]. In this case the qubit states are superpositions of charge states, i.e., $|0\rangle=$ $(|0 e\rangle+|-2 e\rangle) / \sqrt{2}$ and $|1\rangle=(|0 e\rangle-|-2 e\rangle) / \sqrt{2}$, where $|0 e\rangle$ and $|-2 e\rangle$ denote the states with zero and one excess Cooper pair on the island, respectively. Then $Q_{0}^{q} \approx e$ is approximately independent of $\bar{\omega}_{q} \simeq E_{J}$ and we obtain

$$
\zeta=\frac{4 C_{g}^{2}}{C_{r}\left(C_{g}+C_{q}\right)+C_{g}\left(C_{g}+N C_{q}\right)} \times \frac{E_{C}}{E_{J}} .
$$

Therefore, while in practice there might be additional constraints on the achievable parameters, there is no fundamental limit that prevents one from reaching the ultrastrong-coupling regime even for a single qubit, for example, by simply lowering $E_{J}$. This is explicitly shown in Fig. 1(c) for a concrete set of realistic parameters and is in agreement with many previously analyzed circuits $[34,38,39]$ based on the highly nonlinear flux qubit design $[18,40]$.

\section{A. Superradiant charge states}

Given the ability to reach the ultrastrong-coupling regime $g>g_{\text {usc }}$ for a single qubit, it is instructive to develop a physical picture for the ground state in this regime. To do so we consider a semiclassical model $H_{\mathrm{sc}}(\alpha)=\langle\alpha|H| \alpha\rangle$, where $a$ and $a^{\dagger}$ are replaced with the classical field amplitudes $\alpha$ and $\alpha^{*}$. For $N=1$ this model reads

$$
H_{\mathrm{sc}}(\alpha)=\bar{\omega}_{r}|\alpha|^{2}+\frac{\bar{\omega}_{q}}{2} \sigma_{z}+\frac{g}{2}\left(\alpha+\alpha^{*}\right) \sigma_{x} .
$$

In Fig. 2(a) we plot the two eigenenergies $E_{ \pm}(\alpha)$ of $H_{\mathrm{sc}}(\alpha)$, which can be interpreted as Born-Oppenheimer potentials for (a)
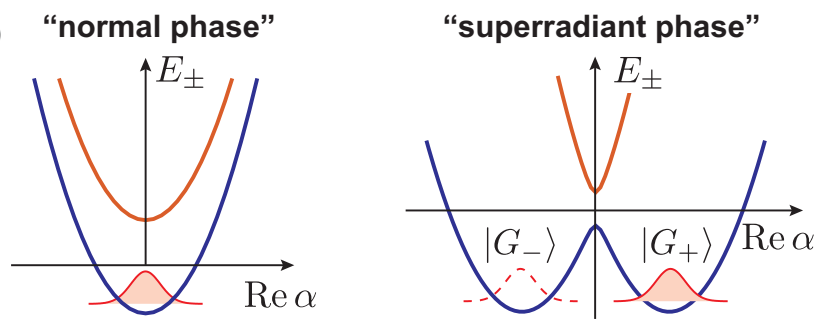

(b)
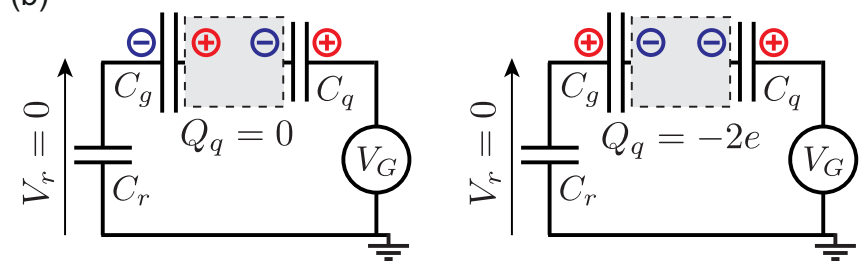

FIG. 2. (a) Plot of the two energy levels $E_{ \pm}(\alpha)$ obtained from the diagonalization of the semiclassical qubit Hamiltonian $H_{\mathrm{sc}}(\alpha)$ for $N=1$. (b) Illustration of the classical charge configurations, which correspond to the two superradiant ground states $\left|G_{ \pm}\right\rangle$.

a classical resonator with amplitude $\alpha$. For $g=0$ the lowest potential curve is simply quadratic, with a minimum at $\alpha=0$. This corresponds to the normal phase, where both the qubit and the resonator state are in the ground state. For values $g \gg g_{\text {usc }}$ the lowest potential curve exhibits two minima, at $\alpha \approx \pm g /\left(2 \bar{\omega}_{r}\right)$. The two essentially degenerate ground states $\left|G_{ \pm}\right\rangle=| \pm \alpha\rangle|\mp\rangle$, where $| \pm\rangle=(|0\rangle \pm|1\rangle) / \sqrt{2}$, then correspond to the superradiant states with a nonvanishing field expectation value.

The physical interpretation of the abstract states $\left|G_{ \pm}\right\rangle$is given in Fig. 2(b), which shows the corresponding classical charge configurations in the limit where all the inductive energies can be neglected. In this limit the charge expectation values for these states are given by $\left\langle G_{ \pm}\left|Q_{r}\right| G_{ \pm}\right\rangle \simeq \pm e$, $\left\langle G_{-}\left|Q_{q}\right| G_{-}\right\rangle \simeq 0$, and $\left\langle G_{+}\left|Q_{q}\right| G_{+}\right\rangle \simeq-2 e$. Most importantly, this simple electrostatic picture clearly illustrates that the main difference in the two configurations is the sign of the polarization charge on the coupling capacitance $C_{g}$. Although the system is in a superradiant state, the voltage across the resonator capacitance $C_{r}$ vanishes. Thus the meaning of a finite field expectation value $\langle a\rangle \neq 0$ in the ground state of a capacitively-coupled circuit QED system is that of a finite polarization charge, while the voltage state of the resonator is unchanged. A closely related observation about the difference between the electric field and the polarization field has previously been pointed out in Ref. [11] for atomic cavity QED systems, but it can be understood here in terms of even simpler electrostatic arguments.

\section{GROUND STATES OF THE EXTENDED DICKE MODEL}

Let us now return to the full circuit QED Hamiltonian (5). Note that for $N \gg 1$ neither Eq. (7) nor Eq. (8) prevents one from reaching the collective ultrastrong-coupling regime, $N \zeta>1$, but now the additional qubit-qubit interactions $\sim D$ must be taken into account. Following the standard approach [5], we first consider the limit $N \gg 1$ and use the Holstein-Primakoff approximation to replace spins with 
harmonic operators, i.e., $S_{z} \rightarrow b^{\dagger} b-N / 2$ and $S_{x} \rightarrow \sqrt{N}(b+$ $\left.b^{\dagger}\right) / 2$, where $\left[b, b^{\dagger}\right]=1$. In this case we obtain the quadratic Hamiltonian (see Appendix B),

$H_{\mathrm{HP}}=\bar{\omega}_{r} a^{\dagger} a+\bar{\omega}_{q} b^{\dagger} b+G\left(a+a^{\dagger}\right)\left(b+b^{\dagger}\right)+D_{N}\left(b+b^{\dagger}\right)^{2}$,

where $G=g \sqrt{N} / 2$ and $D_{N}=N D / 4$. This Hamiltonian can be diagonalized by a Bogoliubov transformation [5,30,31], from which we obtain the two eigenmode frequencies

$$
\omega_{ \pm}^{2}=\frac{1}{2}\left[\bar{\omega}_{r}^{2}+\Omega_{q}^{2} \pm \sqrt{\left(\bar{\omega}_{r}^{2}-\Omega_{q}^{2}\right)^{2}+16 G^{2} \bar{\omega}_{r} \bar{\omega}_{q}}\right]
$$

where $\Omega_{q}^{2}=\bar{\omega}_{q}\left(\bar{\omega}_{q}+4 D_{N}\right)$. The SRT occurs when the ground state of $H_{\mathrm{HP}}$ becomes unstable, i.e., when $\omega_{-}$vanishes. This requires $G^{2}>\bar{\omega}_{c} \Omega_{q} / 4$ or, equivalently, $N\left(g^{2} / \bar{\omega}_{r}\right)>$ $N D+\bar{\omega}_{q}$, and for $D=0$ we recover the usual transition point mentioned above. However, in view of relation (6), the excitation frequencies of the EDM remain positive for all parameter values. This means that in the present circuit QED setup an SRT in the conventional sense does not occur, and it is prevented by a mechanism which is analogous to the effect of polarization interactions discussed, for example, in the context of intersubband polariton systems [30,31]. Since this effect is absent for a single qubit, there is a fundamental difference between single- and multiqubit cavity QED settings, which does not follow from otherwise closely related studies of the $A^{2}$ term $[9,19,20]$.

\section{A. Light-matter decoupling}

Having established the absence of an SRT in the linearized regime, we are now interested in the actual ground state of Hamiltonian (5) under the constraint $D=g^{2} / \bar{\omega}_{r}+\delta$, but for otherwise arbitrary coupling parameters. The inclusion of an additional offset $\delta \geqslant 0$ is motivated by more general circuits discussed in Sec. V. In Figs. 3(a) and 3(b) we plot the expectation values of the mean photon number $\left\langle a^{\dagger} a\right\rangle$ and the spin expectation values $\left\langle S_{z}\right\rangle$ and $\left\langle S_{x}^{2}\right\rangle$ as a function of the coupling $g$ and for $N=9$ and $N=10$ qubits. The plot shows a gradual increase in the photon number, which-as expected from the analysis above-varies smoothly across the SRT point $g=\sqrt{\bar{\omega}_{r} \bar{\omega}_{q} / N}$. This behavior can be fully understood from the linearized Hamilton $H_{\mathrm{HP}}$, from which we derive the approximate initial scaling (see Appendix B)

$$
\left\langle a^{\dagger} a\right\rangle \simeq \frac{N g^{2} \bar{\omega}_{q}}{4\left(\bar{\omega}_{r}+\bar{\omega}_{q}\right)^{2}\left[\bar{\omega}_{q}+N\left(D-g^{2} / \bar{\omega}_{r}\right)\right]} .
$$

The validity of this result requires a low excited-state population of each individual qubit, i.e., $g \ll \bar{\omega}_{r}+\bar{\omega}_{q}$. Beyond this point nonlinear effects start to play a significant role, and surprisingly, for an even number of qubits we observe a sudden decrease in the photon number and $\lim _{g \rightarrow \infty}\left\langle a^{\dagger} a\right\rangle=0$. At the same time the qubits remain in a highly excited state, i.e., $\left\langle S_{z}\right\rangle \approx 0$, with a vanishing polarization along $x$, i.e., $\left\langle S_{x}\right\rangle,\left\langle S_{x}^{2}\right\rangle \rightarrow 0$. In Fig. 3(c) we also plot the entanglement entropy, $\mathcal{S}_{E}(\rho)=-\operatorname{Tr}\left\{\rho \log _{2}(\rho)\right\}$, for the reduced qubit density operator and for the density operator of a single qubit. This shows that while the spin and cavity subsystems become decoupled at large $g$, the qubits remain highly entangled among themselves.
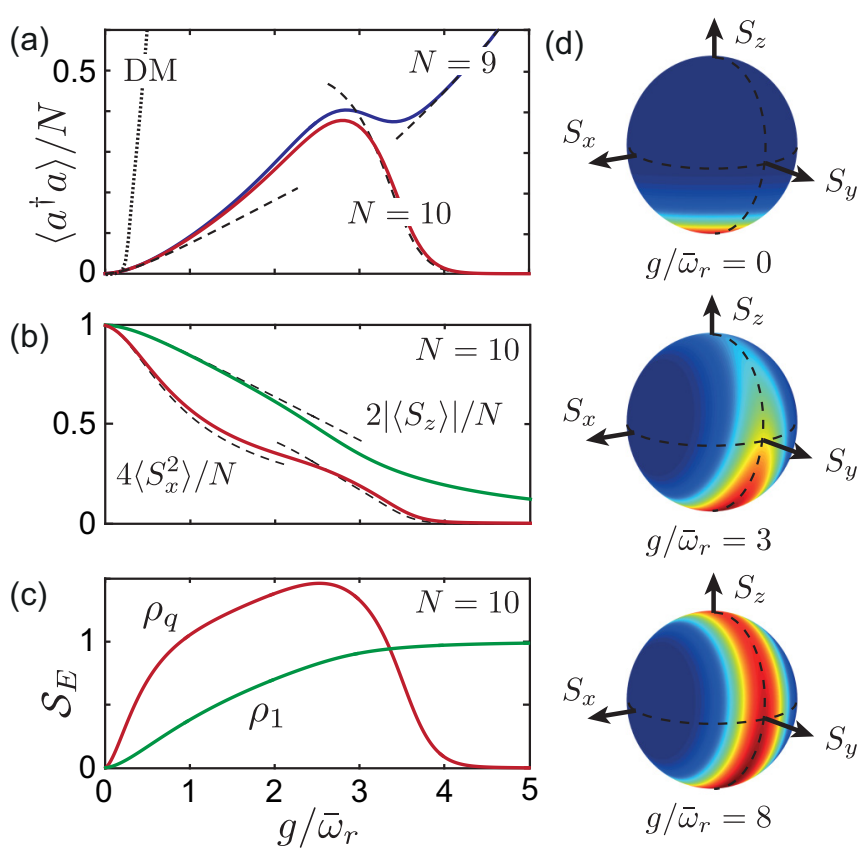

FIG. 3. Ground-state expectation values of (a) the photon number and (b) the collective spin operators as a function of the coupling $g$. The dotted line indicates the prediction from the Dicke model (DM); dashed lines, results obtained from $H_{\mathrm{HP}}$ in Eq. (10) and $H_{\mathrm{eff}}$ in Eq. (15) in the weak- and strong-coupling limit, respectively. (c) Entanglement entropy $\mathcal{S}_{E}(\rho)=-\operatorname{Tr}\left\{\rho \log _{2}(\rho)\right\}$ in the ground state $|G\rangle$ of Hamiltonian (5) evaluated for the reduced density matrix of the qubit subsystem, $\rho_{q}=\operatorname{Tr}_{\mathrm{r}}\{|G\rangle\langle G|\}$, and for the reduced density matrix of a single qubit, $\rho_{1}=\operatorname{Tr}_{N-1}\left\{\rho_{q}\right\}$. (d) Plot of the $Q$ function $Q(\vec{n})=\left\langle\vec{n}\left|\rho_{q}\right| \vec{n}\right\rangle$, where $\vec{n}$ is a unit vector on the Bloch sphere and $|\vec{n}\rangle$ is the corresponding coherent spin state. Note that in (b)-(d) only the results for $N=10$ are shown, and for all plots $\bar{\omega}_{q} / \bar{\omega}_{r}=0.5$ and $\delta=0$ have been assumed.

We remark that other light-matter decoupling mechanisms have recently been described in extended multimode systems $[12,41,42]$. There the inclusion of $A^{2}$-like terms expels the relevant field modes from the coupling region, an effect which can already be described within a linearized model. In the present single-mode setup such a mechanism is not possible, and the above observations already indicate that here the field decoupling is a highly nonlinear and nonclassical effect. A heuristic explanation for this behavior can be obtained by considering the limit $D \gg g, \bar{\omega}_{r, q}$, where the qubit-qubit interaction is the dominant energy and therefore favors the state $\left|m_{x}=0\right\rangle$, where $S_{x}\left|m_{x}\right\rangle=m_{x}\left|m_{x}\right\rangle$, as the ground state. Since the coupling to the field $\sim S_{x}$, it then also vanishes and the resonator mode decouples. This is illustrated in Fig. 3(d), in terms of the $Q$ function of the reduced qubit state on the Bloch sphere, which approaches a circle in the $x=0$ plane for large $g$. For odd $N$, there is no $m_{x}=0$ state, which explains why this decoupling mechanism does not take place for the example of $N=9$ qubits.

\section{B. Effective low-energy model}

Let us now develop a more accurate description of the system in the regime $g / \bar{\omega}_{r}>1$. We write $H=H_{0}+H_{1}$, 


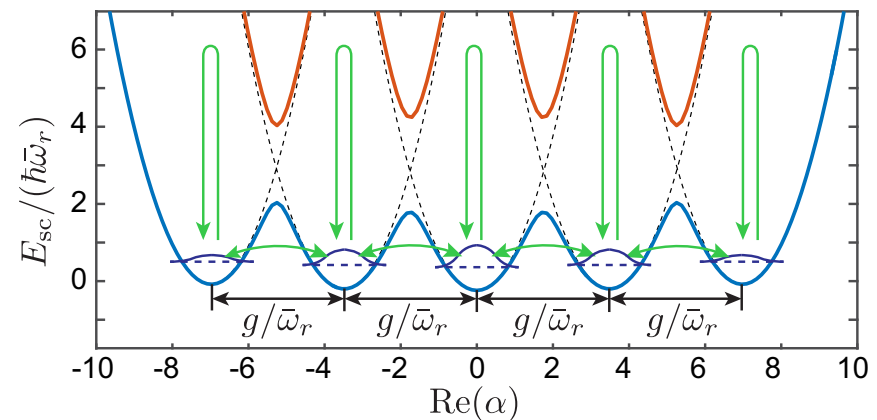

FIG. 4. Plot of the lowest two energy levels $E_{\mathrm{sc}}$ obtained from the diagonalization of the semiclassical qubit Hamiltonian $H_{\mathrm{sc}}(\alpha)=$ $\langle\alpha|H| \alpha\rangle$ and for $g / \bar{\omega}_{r}=3.5, \bar{\omega}_{q} / \bar{\omega}_{r}=1$, and $N=4$. Arrows indicate first- and second-order processes induced by $H_{1}=\bar{\omega}_{q} S_{z}$ on the otherwise degenerate ground-state manifold.

where $H_{1}=\bar{\omega}_{q} S_{z}$. The first term, $H_{0}$, commutes with $S_{x}$ and therefore it can be diagonalized exactly, $H_{0}\left|\Psi_{s, m_{x}, n}\right\rangle=$ $E_{m_{x}, n}\left|\Psi_{s, m_{x}, n}\right\rangle$ (see Appendix C). The eigenstates are given by

$$
\left|\Psi_{s, m_{x}, n}\right\rangle=e^{-\frac{g}{\bar{\omega}_{r}}\left(a^{\dagger}-a\right) S_{x}}\left|s, m_{x}\right\rangle|n\rangle,
$$

where $|n\rangle$ are photon number states and $\left|s, m_{x}\right\rangle$ are collective spin states of given total spin $s=0, \ldots, N / 2$ and projection quantum number $m_{x}=-s, \ldots, s$. Therefore, the eigenstates of $H_{0}$ are simply harmonic oscillator states, which are displaced by the amount $-m_{x} g / \bar{\omega}_{r}$. The corresponding eigenenergies are

$$
E_{m_{x}, n}=\delta m_{x}^{2}+\bar{\omega}_{r} n
$$

which for $\delta \rightarrow 0$ become independent of the spin quantum numbers. This means that in this limit each $n$ manifold contains a set of $2^{N}$ degenerate qubit states. The energy penalty from the $S_{x}^{2}$ term is exactly compensated by a lowering of the interaction energy when the resonator mode is displaced. This is illustrated in Fig. 4, where we plot the lowest two eigenvalues of the semiclassical qubit Hamiltonian, $H_{\mathrm{sc}}(\alpha)$, for $s=N / 2$. Compared to the double-well potential structure for the single-qubit case, the lowest Born-Oppenheimer potential now displays a multiwell potential landscape with $(2 s+1)$ nearly degenerate minima. Again, this large degree of degeneracy can be understood from the corresponding classical charge configurations, as illustrated in Fig. 5 for the case $N=2$. For both qubit configurations, which correspond to different values of $m_{x}$, the electrostatic energy is concentrated only in the gate and coupling capacitances $C_{q}$ and $C_{g}$, and it is therefore identical. Note that the DM with $D=0$ would energetically favor states with $\left|m_{x}\right|=1$ over a $m_{x}=0$ state and lead to inconsistencies with basic electrostatic considerations.

To model the low-energy properties of $H$ we now focus on the ground-state manifold, $n=0$, and include quantum corrections due to $H_{1}$. This term first couples neighboring $m_{x}$ levels within this manifold, i.e., $\left\langle\Psi_{s, m_{x}, 0}\left|S_{z}\right| \Psi_{s, m_{x} \pm 1,0}\right\rangle=$ $e^{-g^{2} / 2 \bar{\omega}_{r}^{2}}\left\langle s, m_{x}\left|S_{z}\right| s, m_{x} \pm 1\right\rangle$. In addition, $H_{1}$ also couples to states in energetically higher manifolds, $n>0$. This effect can be treated in second-order perturbation theory as detailed in Appendix C. These second-order corrections predominantly lead to an energy shift $\sim\left[m_{x}^{2}-s(s+1)\right] \bar{\omega}_{q}^{2} \bar{\omega}_{r} /\left(2 g^{2}\right)$, which singles out the $s=N / 2$ states as the lowest energy manifold.
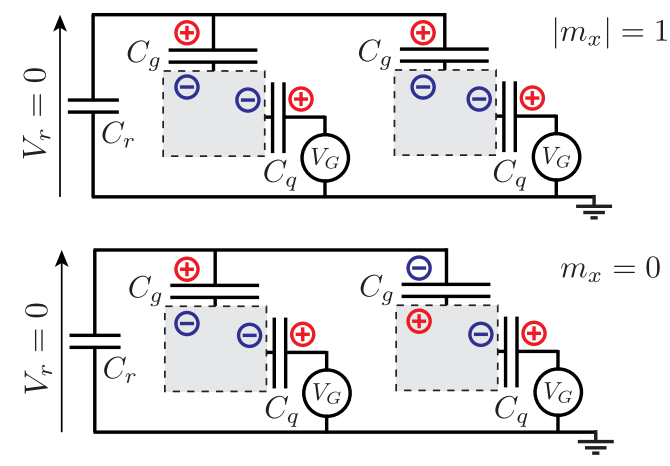

FIG. 5. Illustration of the classical charge configurations, which correspond to two different qubit states with different values of $m_{x}$. In both cases the capacitive energies are the same, which, when generalized to $N$ qubits, explains the existence of $2^{N}$-fold degenerate manifolds in the limit $g \rightarrow \infty$. As in the single-qubit case, the average voltage across the resonator capacitance $C_{r}$ vanishes for all those states.

By defining operators $\tilde{S}_{k}$ via the relation $\left\langle\Psi_{s, m_{x}^{\prime}, 0}\left|\tilde{S}_{k}\right| \Psi_{s, m_{x}, 0}\right\rangle=$ $\left\langle s, m_{x}^{\prime}\left|S_{k}\right| s, m_{x}\right\rangle$, we obtain within this manifold the effective collective spin Hamiltonian

$$
H_{\mathrm{eff}} \simeq\left(\delta+\frac{\bar{\omega}_{q}^{2} \bar{\omega}_{r}}{2 g^{2}}\right) \tilde{S}_{x}^{2}+\bar{\omega}_{q} e^{-g^{2} / 2 \bar{\omega}_{r}^{2}} \tilde{S}_{z} .
$$

From this model we immediately see that for $g / \bar{\omega}_{r} \gg 1$ the coupling to neighboring $m_{x}$ states is exponentially suppressed, and for even $N$ the ground state is indeed $|G\rangle=\left|\Psi_{N / 2, m_{x}=0, n=0}\right\rangle$ with a vanishing photon number. By taking into account first-order corrections from states $\left|\Psi_{N / 2, m_{x}= \pm 1, n=0}\right\rangle$ we obtain the approximate scalings

$$
\left\langle a^{\dagger} a\right\rangle \approx \frac{N(N+2) g^{6}}{2 \bar{\omega}_{r}^{4} \bar{\omega}_{q}^{2}} e^{-g^{2} / \bar{\omega}_{r}^{2}}, \quad \Delta E \approx \frac{\bar{\omega}_{r} \bar{\omega}_{q}^{2}}{2 g^{2}},
$$

where $\Delta E=E_{1}-E_{0}$ is the gap between the ground and the first excited state. Note that this nonexponential closing of the energy gap is very atypical for qubit-resonator models in the ultrastrong-coupling regime. For an odd number of qubits there is no $m_{x}=0$ state and the ground state is $|G\rangle \simeq\left(\left|\Psi_{N / 2, m_{x}=-1 / 2, n=0}\right\rangle+\left|\Psi_{N / 2, m_{x}=1 / 2, n=0}\right\rangle\right) / \sqrt{2}$ and $\left\langle a^{\dagger} a\right\rangle \simeq g^{2} /\left(4 \bar{\omega}_{r}^{2}\right)$ simply increases in the large- $g$ limit. The next-higher state is the corresponding antisymmetric superposition and therefore the energy splitting now exhibits the usual exponential scaling, i.e., $\Delta E \simeq(N+1) \bar{\omega}_{q} e^{-g^{2} / 2 \bar{\omega}_{r}^{2}}$. Although for certain quantities the admixture of higher $n$ levels must be taken into account (see Appendix C), we find that, within its range of validity, $\sqrt{s(s+1)}<g^{2} /\left(\bar{\omega}_{r} \bar{\omega}_{q}\right)$ and $g / \bar{\omega}_{r}>1$, the effective model $H_{\text {eff }}$ provides an accurate description of the low-energy properties of an ultrastrongly coupled collective circuit QED system.

\section{Energy spectrum}

Apart from the above-described changes in the ground-state properties, the appearance of the $D S_{x}^{2}$ term will also change the excitation spectrum of multiqubit circuit QED systems in the ultrastrong-coupling regime. This is exemplified in Fig. 6 


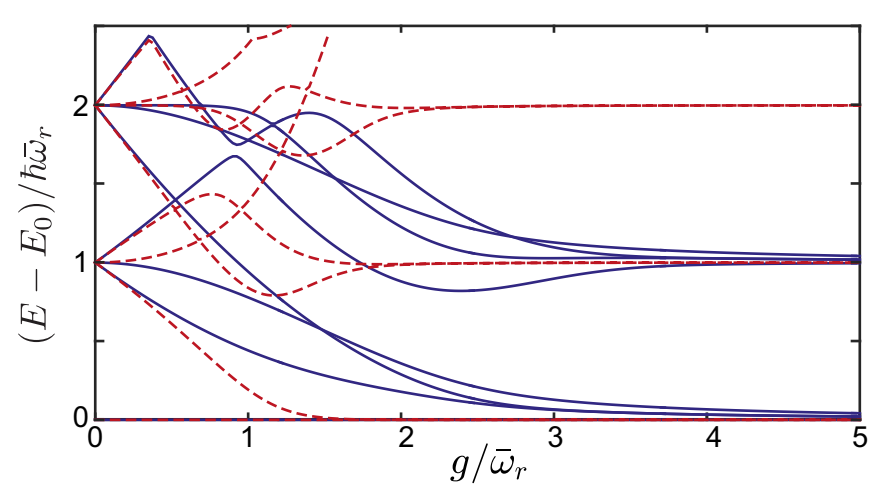

FIG. 6. The lowest excitation energies $\Delta E_{n}=E_{n}-E_{0}$ of the EDM, (5), for $N=2$ qubits are plotted as a function of the coupling strength $g$ and for $\bar{\omega}_{r}=\bar{\omega}_{q}$. Dashed lines show the corresponding excitation energies for the standard DM, where $D=0$.

for the case $N=2$, where the lowest few excitation energies $\Delta E_{n}=E_{n}-E_{0}$ are plotted as a function of the coupling $g$ and compared with the corresponding results for the DM. One clearly sees the emergence of the $2^{N}$-fold degenerate manifolds for large $g$, which already for two qubits is in clear contrast to the twofold degenerate energy manifolds known from the DM. Spectroscopically, the difference between the two models becomes most significant in the region $1<g / \bar{\omega}_{r}<2$, where the crossover from the strong- to the ultrastrong-coupling regime takes place.

\section{FLUX QUBIT CIRCUITS AND EXPERIMENTAL IMPLEMENTATIONS}

The results presented so far have been explicitly derived for a charge coupled circuit, but the appearance of a $D S_{x}^{2}$ term in multiqubit circuits is a more general phenomenon. In Fig. 7(a) we show an equivalent circuit model for $N$ flux qubits coupled inductively to a single $L C$ resonator. The Lagrangian for this

(a)

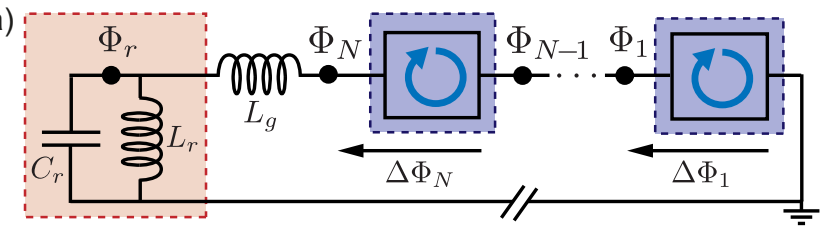

(b)

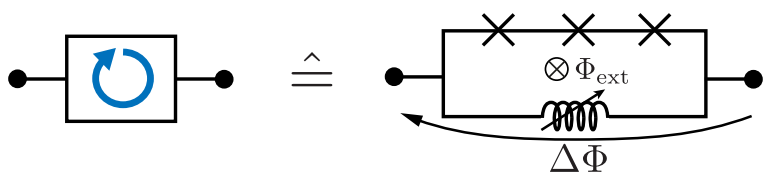

FIG. 7. Circuit model for a collective QED system with inductively coupled flux qubits. Expressed in terms of the flux across each qubit, $\Delta \Phi_{i}=\Phi_{i}-\Phi_{i-1}$, the magnetic energy $\left(\Phi_{r}-\right.$ $\left.\sum \Delta \Phi_{i}\right)^{2} /\left(2 L_{g}\right)$ associated with the inductance $L_{g}$ leads to qubitresonator as well as qubit-qubit interactions, with the relation $D=$ $g^{2} / \bar{\omega}_{r}+\delta$, where $\delta>0$. (b) Specific realization of a flux qubit based on the design used in Refs. $[25,26]$ to reach the ultrastrong-coupling regime. circuit is

$$
\mathcal{L}=\frac{C_{r} \dot{\Phi}_{r}^{2}}{2}-\frac{\Phi_{r}^{2}}{2 L_{r}}-\frac{\left(\Phi_{r}-\Phi_{N}\right)^{2}}{2 L_{g}}+\sum_{i=1}^{N} \mathcal{L}_{q}\left(\Delta \Phi_{i}, \Delta \dot{\Phi}_{i}\right)
$$

Here $\mathcal{L}_{q}$ denotes the Lagrangian for a single flux qubit, which is a function of the phase difference $\Delta \Phi_{i}=\Phi_{i}-\Phi_{i-1}$ and, depending on the exact design, of additional local degrees of freedom [18,34,38-40]. Since for this circuit there are no capacitive couplings between the individual components, the derivation of the corresponding circuit Hamiltonian can be performed in a straightforward manner. In particular, by writing $\Phi_{N}=\sum_{i} \Delta \Phi_{i}$, one immediately sees that the resulting inductive interaction between $\Phi_{r}$ and $\Phi_{N}$ can be grouped as

$$
\frac{\left(\Phi_{r}-\Phi_{N}\right)^{2}}{2 L_{g}}=\frac{\Phi_{r}^{2}}{2 L_{g}}-\sum_{i} \frac{\Delta \Phi_{i} \Phi_{r}}{L_{g}}+\sum_{i, j} \frac{\Delta \Phi_{i} \Delta \Phi_{j}}{2 L_{g}}
$$

The first $A^{2}$-like term leads to a renormalization of the resonator inductance $L_{r} \rightarrow \bar{L}_{r}=\left(L_{r} L_{g}\right) /\left(L_{r}+L_{g}\right)$, which, however, does not scale with $N$. The other two terms represent the collective qubit-photon coupling and the collective qubit-qubit interactions, respectively. By writing $\Phi_{r}=$ $\sqrt{\hbar / 2 C_{r} \bar{\omega}_{r}}\left(a^{\dagger}+a\right)$, and within the validity of the two-level approximation, i.e., $\Delta \Phi_{i}=\Phi_{q}^{0} \sigma_{x}^{i}$, we recover the EDM, (5), with the more general relation

$$
D=\frac{g^{2}}{\bar{\omega}_{r}}\left(1+\frac{L_{g}}{L_{r}}\right)=\frac{g^{2}}{\bar{\omega}_{r}}+\delta,
$$

where $\delta>0$, as assumed in the analysis above.

The flux qubit circuit shown in Fig. 7(a) is also very promising for first experimental realizations of the described models. Compared to capacitive interactions, where $g / \bar{\omega}_{r} \sim$ $\sqrt{\bar{Z}_{r} / R_{K}}\left[R_{K}=h /\left(e^{2}\right)\right.$ is the quantum resistance $]$, the inductive coupling $g / \bar{\omega}_{r} \sim \sqrt{R_{K} / \bar{Z}_{r}}$ scales more favorably with the resonator impedance $\bar{Z}_{r}=\bar{L}_{r} / \bar{C}_{r}$ [17]. Figure 7(b) shows a sketch of a three-junction flux qubit with an additional tunable inductance. This design has been used in recent experiments to demonstrate ultrastrong-coupling conditions in a single-mode setup [25] as well as for an open transmission line [26] and exhibits a large degree of tunability. The addition of a second qubit in such setups would already allow the observation of the described decoupling effect, either by looking at the excitation spectrum shown in Fig. 6 or by measuring the predicted anticorrelations of the qubit flux states in the ground state of the system.

\section{A. Other experimental considerations}

In our analysis so far we have assumed identical couplings $g_{i}=g$ and identical qubit frequencies $\bar{\omega}_{q}^{i}=\bar{\omega}_{q}$, which can be hard to realize in practice $[43,44]$. However, in Fig. 8 we reevaluate the light-matter decoupling effect for a disordered system, where we allow for individual variations of the $g_{i}$ and $\bar{\omega}_{q}^{i}$. We see that even at a very high level of disorder there are small quantitative differences, but almost no qualitative changes of the predicted features. Surprisingly, even the parity oscillations, which we explained above in terms of a fully symmetric coupling, are very robust with respect to variations 
(a)

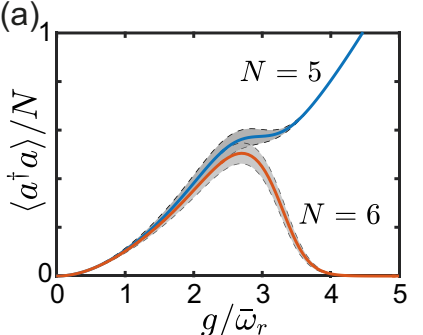

(c)

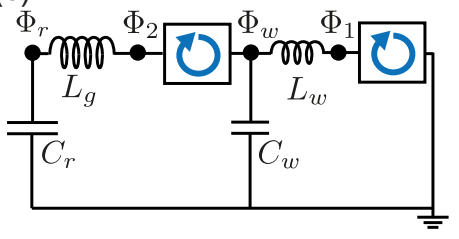

(b)

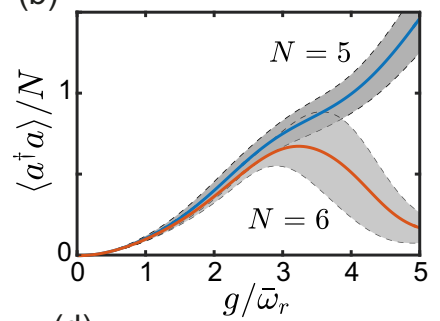

(d)

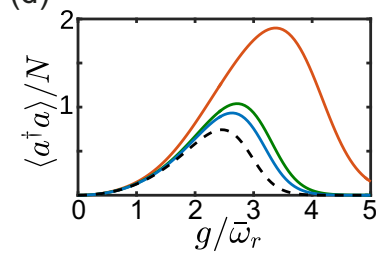

FIG. 8. (a) The ground-state photon number $\left\langle a^{\dagger} a\right\rangle$ is plotted for a disordered system, where the qubit frequencies $\bar{\omega}_{q}^{i}$ are randomly chosen from a uniform distribution in the interval $\left[\bar{\omega}_{q}-0.5 \bar{\omega}_{q}, \bar{\omega}_{q}+\right.$ $\left.0.5 \bar{\omega}_{q}\right]$. (b) The same as (a), but with a random distribution of coupling constants $g_{i}$ chosen from the interval $[g-0.3 g, g+0.3 g]$. Other parameters for this plot are the same as in Fig. 3. (c) Circuit model for two flux qubits, which are connected via a wire of finite inductance $L_{w}$ and capacitance $C_{w}$. The frequencies $\bar{\omega}_{r}$ and $\omega_{\mathrm{ex}}$ are identified with the lower and higher resonator frequencies and $g$ is identified with the coupling of the first qubit with the lower resonator mode. Other coupling constants are identified as $g_{i k}$, where $i=1,2$ stands for the qubits, $k=1,2$ for the modes, and $k=1$ is the lower and $k=2$ the higher. Thus, under this naming convention $g \equiv g_{11}$. All of the parameters can be derived from the circuit by diagonalizing the harmonic part of the circuit's Hamiltonian. (d) Ground-state photon number in the presence of a higher mode. For the plot we use $L_{w}=L_{g} / 3, C_{w}=C_{r} / 87, \omega_{\mathrm{ex}} / \bar{\omega}_{r}=21.56, g_{12} / g=2.69$, $g_{21} / g=1.00, g_{22} / g=-8.04$ (blue curve), $L_{w}=L_{g}, C_{w}=C_{r} / 29$, $\omega_{\text {ex }} / \bar{\omega}_{r}=10.86, g_{12} / g=3.35, g_{21} / g=1.02, g_{22} / g=-3.30($ green curve), and $L_{w}=3 L_{g}, C_{w}=3 C_{r} / 29, \omega_{\mathrm{ex}} / \bar{\omega}_{r}=7.61, g_{12} / g=5.15$, $g_{21} / g=1.08, g_{22} / g=-1.59$ (red curve). For all curves we have chosen $L_{g}=1.5 \mathrm{nH}$ and $C_{r}=0.25 \mathrm{pF}$. The dotted line displays the single-mode result. Values for $L_{w}$ and $C_{w}$ in the three curves correspond to an $\sim 200-\mu \mathrm{m}$-long (blue curve), $\sim 500-\mu \mathrm{m}$-long (green curve,) and $\sim 1$-mm-long (red curve) wire between the qubits. For all curves qubit parameters have been adjusted to give the same frequency, $\bar{\omega}_{q}=\bar{\omega}_{r} / 2$.

in the coupling constants and all curves are still clearly distinct from the sharp increase in $\left\langle a^{\dagger} a\right\rangle$ expected from the DM. Therefore, the predicted effects should be observable even in systems with only a limited amount of tunability.

Finally, our model assumes the coupling of all qubits to a single resonator mode. This is in general a good approximation for lumped element resonators, where the fundamental electromagnetic mode can be well separated from all higher excitations. For example, considering a typical $L C$ resonator of spatial extent $d \sim 500 \mu \mathrm{m}$ and a fundamental mode of $\bar{\omega}_{r} /(2 \pi) \sim 5 \mathrm{GHz}[23,25]$ one expects higher order modes at a frequency $\omega_{\mathrm{ex}} /(2 \pi) \sim c /(6 d) \sim 100 \mathrm{GHz}$ [46]. At the same time such a circuit can incorporate tens of flux qubits of size $\sim 10 \mu \mathrm{m}$. To understand the validity of the single-mode approximation more quantitatively, we investigate the twomode setup shown in Fig. 8(c), where the ideal wire connecting

two flux qubits is replaced with a finite inductance $L_{w}$ and capacitance $C_{w}$. This circuit is used to model a higher excited mode with frequency $\omega_{\mathrm{ex}} \sim 1 / \sqrt{L_{w} C_{w}}$. Figure 8 (d) shows the dependence of the photon number $\left\langle a^{\dagger} a\right\rangle$ in the ground state for varying ratios $\omega_{\mathrm{ex}} / \bar{\omega}_{r}$. Again, we observe a robustness of the decoupling effect, and for the experimentally relevant regime $\omega_{\mathrm{ex}} / \bar{\omega}_{r} \geqslant 20$ (corresponding to an $\sim 200-\mu \mathrm{m}$-long wire) there is no significant influence of the higher mode. This ability to realize a single-mode setup in the ultrastrong-coupling regime is also one of the key advantages of circuit QED, since such a separation of modes cannot be achieved in the optical regime.

\section{CONCLUSIONS}

In summary we have analyzed collective interactions in circuit QED systems in the ultrastrong-coupling regime. Going beyond the previously discussed $A^{2}$ corrections, we have identified the important role of qubit-qubit interactions, which generically appear in the fundamental description of such circuits. In particular, we have shown that the ground state of the resulting EDM exhibits many features that are exactly opposite to what is expected from the often considered DM physics. Apart from the absence of a superradiant phase, this includes light-matter decoupling at very strong interactions, a high degree of entanglement between the qubits and the existence of manifolds with an exponentially large number of nearly degenerate states. These predictions can already be tested with a minimal setup consisting of two flux qubits coupled to a lumped element resonator, similar to existing experimental setups $[25,26]$.

On a broader scope, we have presented in this work a fully microscopic derivation of a minimal model describing multiple (artificial) atoms coupled to a single radiation mode, where, compared to optical systems, the single-mode or two-level approximations can be rigorously justified by an appropriate circuit design. The resulting model is identical to the single-mode Hopfield model, which is usually derived for macroscopic dielectrics and has been successfully applied to describe collective ultrastrong-coupling effects in solid-state cavity QED systems [30]. Our analysis reveals for the first time the highly nontrivial quantum mechanical features of this fundamental model in the regime where each individual atom is coupled ultrastrongly to the radiation field.

\section{ACKNOWLEDGMENTS}

We thank J. Majer and S. Putz for stimulating discussions. This work was supported by the Austrian Science Fund (FWF) through SFB FOQUS F40, DK CoQuS W 1210, and START Grant Y 591-N16 and by the European Commission through FP7/ITC Project SIQS (600645) and Marie Sklodowska-Curie Grant IF 657788. J.J.G.R. acknowledges support from Spanish Mineco Project FIS2012-33022 and the CAM Research Network QUITEMAD+.

\section{APPENDIX A: CIRCUIT QED HAMILTONIAN}

In this Appendix we provide additional details on the derivation of the charge Hamiltonian, (3). To perform the Legendre transformation we write the circuit Lagrangian, (1), 
as

$$
\mathcal{L}=\frac{1}{2} \dot{\boldsymbol{\Phi}}^{\mathrm{T}} \mathcal{C} \dot{\boldsymbol{\Phi}}-\dot{\boldsymbol{\Phi}}^{\mathrm{T}} \boldsymbol{a}+\frac{N C_{q} V_{G}^{2}}{2}-V_{\mathrm{pot}}\left(\left\{\Phi_{\eta}\right\}\right),
$$

where $\dot{\boldsymbol{\Phi}}=\left(\dot{\Phi}_{r}, \dot{\Phi}_{1}, \ldots, \dot{\Phi}_{N}\right)^{T}, V_{\text {pot }}\left(\left\{\Phi_{\eta}\right\}\right)$ is the potential energy, and $\boldsymbol{a}=\left(0, C_{q} V_{G}, \ldots, C_{q} V_{G}\right)^{T}$. For the circuit shown in Fig. 1(a) the capacitance matrix is

$\mathcal{C}=\left(\begin{array}{ccccc}C_{r}+N C_{g} & -C_{g} & -C_{g} & \ldots & -C_{g} \\ -C_{g} & C_{q}+C_{g} & 0 & \ldots & 0 \\ -C_{g} & 0 & C_{q}+C_{g} & \ldots & 0 \\ \vdots & \vdots & \vdots & \ddots & \vdots \\ -C_{g} & 0 & 0 & \ldots & C_{q}+C_{g}\end{array}\right)$.

The Hamiltonian function is obtained from the Lagrangian via a Legendre transformation and can be written in vector notation as

$$
\mathcal{H}=\frac{1}{2}(\boldsymbol{Q}+\boldsymbol{a})^{T} \mathcal{C}^{-1}(\boldsymbol{Q}+\boldsymbol{a})+V_{\mathrm{pot}}\left(\left\{\Phi_{\eta}\right\}\right),
$$

where $\boldsymbol{Q}=\left(Q_{r}, Q_{1}, \ldots, Q_{N}\right)^{T}$ is the vector of conjugate charges $\boldsymbol{Q}=\partial \mathcal{L} / \partial \dot{\boldsymbol{\Phi}}$. The inverse of the capacitance matrix is of the form

$$
\frac{1}{\mathcal{C}}=\frac{1}{\bar{C}^{2}}\left(\begin{array}{ccccc}
C_{q}+C_{g} & C_{g} & C_{g} & \ldots & C_{g} \\
C_{g} & X & Y & \ldots & Y \\
C_{g} & Y & X & \ldots & Y \\
\vdots & \vdots & \vdots & \ddots & \vdots \\
C_{g} & Y & Y & \ldots & X
\end{array}\right)
$$

where $\bar{C}^{2}=C_{r} C_{q}+C_{g}\left(C_{r}+N C_{q}\right)$ and we have used the abbreviations $X=C_{r}+C_{g}+(N-1) C_{g} C_{q} /\left(C_{g}+C_{q}\right), Y=$ $C_{g}^{2} /\left(C_{g}+C_{q}\right)$. The result for the charge Hamiltonian, (3), then follows directly from Eqs. (A3) and (A4).

\section{APPENDIX B: HOLSTEIN-PRIMAKOFF APPROXIMATION}

The Holstein-Primakoff approximation is based on an exact mapping of collective spin operators $S_{ \pm}=S_{x} \pm i S_{y}$ and $S_{z}$ onto a bosonic mode with annihilation operator $b[5,45]$ :

$$
\begin{aligned}
& S_{+}=b^{\dagger} \sqrt{N-b^{\dagger} b}, \\
& S_{-}=\sqrt{N-b^{\dagger} b} b, \\
& S_{z}=\left(b^{\dagger} b-\frac{N}{2}\right) .
\end{aligned}
$$

Under the assumption that $N \gg 1$ and the total number of excitations remains small, i.e., $\left\langle b^{\dagger} b\right\rangle / N \ll 1$, we can approximate $S_{x} \simeq \sqrt{N}\left(b^{\dagger}+b\right) / 2$ in the EDM, (5), and we obtain the quadratic Hamiltonian $H_{\mathrm{HP}}$ given in Eq. (10). This Hamiltonian can be diagonalized and written as [5]

$$
H_{\mathrm{HP}}=\sum_{\alpha= \pm} \hbar \omega_{\alpha} c_{\alpha}^{\dagger} c_{\alpha}
$$

where the excitation frequencies are given by

$$
\omega_{ \pm}^{2}=\frac{1}{2}\left[\bar{\omega}_{r}^{2}+\Omega_{q}^{2} \pm \sqrt{\left(\bar{\omega}_{r}^{2}-\Omega_{q}^{2}\right)^{2}+16 G^{2} \bar{\omega}_{r} \bar{\omega}_{q}}\right]
$$

and $\Omega_{q}^{2}=\bar{\omega}_{q}\left(\bar{\omega}_{q}+4 D_{N}\right)$. By using the relation $D=g^{2} / \bar{\omega}_{r}+$ $\delta$ we find that both excitation frequencies are positive as long as $\delta \geqslant 0$ and $\bar{\omega}_{q}>0$.

The ground state $|G\rangle$ of this Hamiltonian is characterized by $c_{ \pm}|G\rangle=0$. Using this property we can calculate the average photon number in the ground state. We obtain

$$
\left\langle a^{\dagger} a\right\rangle=\frac{\cos (2 \theta) A_{-}+A_{+}-4}{8},
$$

where we have used the short-hand notation

$$
\begin{gathered}
\cos (2 \theta)=\frac{\bar{\omega}_{r}^{2}-\Omega_{q}^{2}}{\sqrt{\left(\bar{\omega}_{r}^{2}-\Omega_{q}^{2}\right)^{2}+16 G^{2} \bar{\omega}_{c} \bar{\omega}_{q}}}, \\
A_{ \pm}=\frac{\left(\omega_{+} \omega_{-} \pm \bar{\omega}_{r}^{2}\right)\left(\omega_{+} \pm \omega_{-}\right)}{\bar{\omega}_{r} \omega_{+} \omega_{-}} .
\end{gathered}
$$

For small $g$ the result for $\left\langle a^{\dagger} a\right\rangle$ can be further simplified to the expression given in Eq. (12) in the text.

\section{APPENDIX C: ULTRASTRONG-COUPLING PERTURBATION THEORY}

In this Appendix we summarize the details of the perturbation theory used to describe the ground-state properties in the ultrastrong-coupling regime $g>\bar{\omega}_{r}, \bar{\omega}_{q}$. In this regime the full Hamiltonian can be written as $H=H_{0}+H_{1}$, where

$$
H_{0}=\hbar \bar{\omega}_{r} a^{\dagger} a+\hbar g\left(a^{\dagger}+a\right) S_{x}+\hbar D S_{x}^{2}
$$

and $H_{1}=\bar{\omega}_{q} S_{z}$. We see that $H_{0}$ commutes with $S_{x}$ and it can be diagonalized by a polaron transformation, $H_{0}^{\prime}=U^{\dagger} H_{0} U$, where $U=\exp \left(-\gamma\left(a^{\dagger}-a\right) S_{x}\right)$ and $\gamma=g / \bar{\omega}_{r}$. We obtain

$$
H_{0}^{\prime}=\hbar \bar{\omega}_{r} a^{\dagger} a+\hbar \underbrace{\left(D-\frac{g^{2}}{\bar{\omega}_{r}}\right)}_{\delta} S_{x}^{2} .
$$

Therefore, in this new frame the eigenstates are $\left|s, m_{x}\right\rangle|n\rangle$, where $s$ is the total spin quantum number, $m_{x}$ is the spin projection along $x$, and $|n\rangle$ is the number state of the resonator mode. The corresponding energies are $E_{m_{x}, n}=\hbar \bar{\omega}_{r} n+\hbar \delta m_{x}^{2}$. After transforming back into the original frame we obtain the eigenstates $\left|\Psi_{s, m_{x}, n}\right\rangle$ defined in Eq. (13).

For $\delta \rightarrow 0$ the eigenspectrum of $H_{0}$ consists of a set of highly degenerate manifolds separated by multiples of $\hbar \bar{\omega}_{r}$. This degeneracy is lifted by the qubit Hamiltonian $H_{1}$ and in the following we are interested in the effect of $H_{1}$ on the ground-state manifold spanned by the states with $n=0$. To do so we need the matrix elements

$$
\begin{aligned}
& \left\langle\Psi_{s, m_{x}^{\prime}, n}\left|H_{1}\right| \Psi_{s, m_{x}, 0}\right\rangle \\
& =\hbar \bar{\omega}_{q} e^{-\frac{\gamma^{2}\left(m_{x}-m_{x}^{\prime}\right)^{2}}{2}} \frac{\gamma^{n}\left(m_{x}^{\prime}-m_{x}\right)^{n}}{\sqrt{n !}}\left\langle s, m_{x}^{\prime}\left|S_{z}\right| s, m_{x}\right\rangle .
\end{aligned}
$$

To first order in $H_{1}$ we obtain a tunneling between neighboring $m_{x}$ states within the $n=0$ manifold, i.e.,

$$
\left\langle\Psi_{s, m_{x}^{\prime}, 0}\left|H_{1}\right| \Psi_{s, m_{x}, 0}\right\rangle=\hbar \bar{\omega}_{q} e^{-\frac{\gamma^{2}}{2}}\left\langle s, m_{x}^{\prime}\left|S_{z}\right| s, m_{x}\right\rangle
$$


In terms of the effective spin operators $\tilde{S}_{k}$ we can write the firstorder correction to the effective ground-state Hamiltonian as

$$
H_{\mathrm{eff}}^{(1)}=\hbar \bar{\omega}_{q} e^{-\frac{\gamma^{2}}{2}} \tilde{S}_{z} .
$$

To second order in $\bar{\omega}_{q}$ the states in the ground-state manifold are coupled to higher $n$ states, which are separated by an energy $\hbar \bar{\omega}_{r} n$. These processes can be treated in second-order perturbation theory and we obtain

$$
H_{\mathrm{eff}}^{(2)}=\sum_{s=0}^{N / 2} \sum_{m_{x}, m_{x}^{\prime}=-s}^{s} M\left(s, m_{x}^{\prime}, m_{x}\right)\left|\Psi_{s, m_{x}^{\prime}, 0}\right\rangle\left\langle\Psi_{s, m_{x}, 0}\right|,
$$

where

$$
\begin{aligned}
M\left(s, m_{x}^{\prime}, m_{x}\right) & \\
= & -\sum_{n=1}^{\infty} \frac{\left\langle\Psi_{s, m_{x}^{\prime}, 0}\left|H_{1}\right| \Psi_{s, m_{x}+1, n}\right\rangle\left\langle\Psi_{s, m_{x}+1, n}\left|H_{1}\right| \Psi_{s, m_{x}, 0}\right\rangle}{\left(2 m_{x}+1\right) \hbar \delta+\hbar \bar{\omega}_{r} n} \\
& -\sum_{n=1}^{\infty} \frac{\left\langle\Psi_{s, m_{x}^{\prime}, 0}\left|H_{1}\right| \Psi_{s, m_{x}-1, n}\right\rangle\left\langle\Psi_{s, m_{x}-1, n}\left|H_{1}\right| \Psi_{s, m_{x}, 0}\right\rangle}{\left(-2 m_{x}+1\right) \hbar \delta+\hbar \bar{\omega}_{r} n}
\end{aligned}
$$

As a main contribution we obtain a diagonal term, which for small $\delta$ and $\gamma \gtrsim 2$ is approximately given by

$$
\begin{aligned}
M\left(s, m_{x}, m_{x}\right) & =\frac{\hbar \bar{\omega}_{q}^{2}}{2 \bar{\omega}_{r}}\left[m_{x}^{2}-s(s+1)\right] e^{-\gamma^{2}} \sum_{n=1}^{\infty} \frac{\gamma^{2 n}}{n ! n} \\
& \simeq \frac{\hbar \bar{\omega}_{q}^{2}}{2 \bar{\omega}_{r} \gamma^{2}}\left[m_{x}^{2}-s(s+1)\right],
\end{aligned}
$$

and we obtain the second-order contribution

$$
H_{\mathrm{eff}}^{(2)}=\frac{\hbar \bar{\omega}_{q}^{2} \bar{\omega}_{r}}{2 g^{2}}\left[\tilde{S}_{x}^{2}-\overrightarrow{\tilde{S}}^{2}\right]
$$

Since these energy shifts are not exponentially suppressed, $H_{\mathrm{eff}}^{(2)}$ dominates over $H_{\mathrm{eff}}^{(1)}$ and determines the basic ordering of the energy levels for $g \rightarrow \infty$. In particular, this result shows that for given $m_{x}$ the maximal angular momentum state with $s=N / 2$ is lowest in energy. Since $H$ preserves the total angular momentum, it is enough to evaluate the low excitation properties within this $s=N / 2$ manifold.

Note that, in addition to the energy correction, we obtain a correction to the state vectors. For most quantities these corrections are not essential, but they can lead to additional contributions in the expectation values, which are not taken into account in the analysis in the text. A significant correction occurs, for example, for the qubit polarization $\left\langle\Psi_{N / 2,0,0}\left|S_{z}\right| \Psi_{N / 2,0,0}\right\rangle$, which, according to $H_{\text {eff }}$, would decay exponentially at large $g$ but, in reality, decays algebraically, i.e., $\left\langle\Psi_{N / 2,0,0}\left|S_{z}\right| \Psi_{N / 2,0,0}\right\rangle \approx-N(N+2) \bar{\omega}_{q} \bar{\omega}_{r} /\left(4 g^{2}\right)$.

Finally, we emphasize that the validity of the effective Hamiltonian $H_{\text {eff }}$ requires that for each $n$ the matrix elements given in Eq. (C3) are small compared to the energy difference $\hbar \bar{\omega}_{r} n$. The matrix elements are exponentially suppressed for small $n$ and reach maximal value for $n_{0} \approx \gamma^{2}$. Therefore, the validity of the perturbation theory is restricted to parameters

$$
\frac{\sqrt{s(s+1)} \bar{\omega}_{q}}{2}<\frac{g^{2}}{\bar{\omega}_{r}} .
$$

[1] R. H. Dicke, Coherence in spontaneous radiation processes, Phys. Rev. 93, 99 (1954).

[2] T. Brandes, Coherent and collective quantum optical effects in mesoscopic systems, Phys. Rep. 408, 315 (2005).

[3] K. Hepp and E. H. Lieb, On the superradiant phase transition for molecules in a quantized radiation field: The Dicke Maser model, Ann. Phys. 76, 360 (1973).

[4] Y. K. Wang and F. T. Hioe, Phase transition in the Dicke model of superradiance, Phys. Rev. A 7, 831 (1973).

[5] C. Emary and T. Brandes, Chaos and the quantum phase transition in the Dicke model, Phys. Rev. E 67, 066203 (2003).

[6] K. Baumann, C. Guerlin, F. Brennecke, and T. Esslinger, Dicke quantum phase transition with a superfluid gas in an optical cavity, Nature 464, 1301 (2010).

[7] M. P. Baden, K. J. Arnold, A. L. Grimsmo, S. Parkins, and M. D. Barrett, Realization of the Dicke Model Using CavityAssisted Raman Transitions, Phys. Rev. Lett. 113, 020408 (2014).

[8] J. Klinder, H. Keßler, M. Wolke, L. Mathey, and A. Hemmerich, Dynamical phase transition in the open Dicke model, Proc. Natl. Acad. Sci. USA 112, 3290 (2015).

[9] K. Rzazewski, K. Wodkiewicz, and W. Zakowicz, Phase Transitions, Two-Level Atoms, and the $A^{2}$ Term, Phys. Rev. Lett. 35, 432 (1975).
[10] J. M. Knight, Y. Aharonov, and G. T. C. Hsieh, Are superradiant phase transitions possible? Phys. Rev. A 17, 1454 (1978).

[11] J. Keeling, Coulomb interactions, gauge invariance, and phase transitions of the Dicke model, J. Phys.: Condens. Matter 19, 295213 (2007).

[12] S. De Liberato, Light-Matter Decoupling in the Deep Strong Coupling Regime: The Breakdown of the Purcell Effect, Phys. Rev. Lett. 112, 016401 (2014).

[13] A. Vukics, T. Griesser, and P. Domokos, Elimination of the A-Square Problem from Cavity QED, Phys. Rev. Lett. 112, 073601 (2014).

[14] T. Grießer, A. Vukics, and P. Domokos, Depolarization shift of the superradiant phase transition, Phys. Rev. A 94, 033815 (2016).

[15] A. Blais, R. S. Huang, A. Wallraff, S. M. Girvin, and R. J. Schoelkopf, Cavity quantum electrodynamics for superconducting electrical circuits: An architecture for quantum computation, Phys. Rev. A 69, 062320 (2004).

[16] A. Wallraff, D. I. Schuster, A. Blais, L. Frunzio, R. S. Huang, J. Majer, S. Kumar, S. M. Girvin, and R. J. Schoelkopf, Strong coupling of a single photon to a superconducting qubit using circuit quantum electrodynamics, Nature (London) 431, 162 (2004). 
[17] M. H. Devoret, S. Girvin, and R. Schoelkopf, Circuit-QED: How strong can the coupling between a Josephson junction atom and a transmission line resonator be? Ann. Phys. (NY) 16, 767 (2007).

[18] J. Q. You and F. Nori, Atomic physics and quantum optics using superconducting circuits, Nature (London) 474, 589 (2011).

[19] P. Nataf and C. Ciuti, No-go theorem for superradiant quantum phase transitions in cavity QED and counter-example in circuit QED, Nat. Commun. 1, 72 (2010).

[20] O. Viehmann, J. von Delft, and F. Marquardt, Superradiant Phase Transitions and the Standard Description of Circuit QED, Phys. Rev. Lett. 107, 113602 (2011).

[21] M. Leib and M. J. Hartmann, Synchronized Switching in a Josephson Junction Crystal, Phys. Rev. Lett. 112, 223603 (2014).

[22] T. Niemczyk et al., Circuit quantum electrodynamics in the ultrastrong-coupling regime, Nat. Phys. 6, 772 (2010).

[23] P. Forn-Diaz, J. Lisenfeld, D. Marcos, J. J. Garcia-Ripoll, E. Solano, C. J. P. M. Harmans, and J. E. Mooij, Observation of the Bloch-Siegert Shift in a Qubit-Oscillator System in the Ultrastrong Coupling Regime, Phys. Rev. Lett. 105, 237001 (2010).

[24] Z. Chen, Y. Wang, T. Li, L. Tian, Y. Qiu, K. Inomata, F. Yoshihara, S. Han, F. Nori, J. S. Tsai, and J. Q. You, Multi-photon sideband transitions in an ultrastrongly-coupled circuit quantum electrodynamics system, arXiv:1602.01584.

[25] F. Yoshihara, T. Fuse, S. Ashhab, K. Kakuyanagi, S. Saito, and K. Semba, Superconducting qubit-oscillator circuit beyond the ultrastrong-coupling regime, arXiv:1602.00415.

[26] P. Forn-Diaz, J. J. Garcia-Ripoll, B. Peropadre, M. A. Yurtalan, J.-L. Orgiazzi, R. Belyansky, C. M. Wilson, and A. Lupascu, Ultrastrong coupling of a single artificial atom to an electromagnetic continuum, arXiv:1602.00416.

[27] G. Chen, Z. Chen, and J. Liang, Simulation of the superradiant quantum phase transition in the superconducting charge qubits inside a cavity, Phys. Rev. A 76, 055803 (2007).

[28] N. Lambert, Y.-N. Chen, R. Johansson, and F. Nori, Quantum chaos and critical behavior on a chip, Phys. Rev. B 80, 165308 (2009).

[29] J. J. Hopfield, Theory of the contribution of excitons to the complex dielectric constant of crystals, Phys. Rev. 112, 1555 (1958).

[30] Y. Todorov, A. M. Andrews, R. Colombelli, S. De Liberato, C. Ciuti, P. Klang, G. Strasser, and C. Sirtori, Ultrastrong LightMatter Coupling Regime with Polariton Dots, Phys. Rev. Lett. 105, 196402 (2010).

[31] Y. Todorov and C. Sirtori, Few-Electron Ultrastrong LightMatter Coupling in a Quantum LC Circuit, Phys. Rev. X 4, 041031 (2014).
[32] A. Cottet, T. Kontos, and B. Doucot, Electron-photon coupling in mesoscopic quantum electrodynamics, Phys. Rev. B 91, 205417 (2015).

[33] M. Bamba, K. Inomata, and Y. Nakamura, Superradiant phase transition in superconducting circuit in thermal equilibrium, arXiv:1605.01124.

[34] B. Peropadre, D. Zueco, D. Porras, and J. J. Garcia-Ripoll, Nonequilibrium and Nonperturbative Dynamics of Ultrastrong Coupling in Open Lines, Phys. Rev. Lett. 111, 243602 (2013).

[35] Y. Makhlin, G. Schön, and A. Shnirman, Quantum-state engineering with Josephson-junction devices, Rev. Mod. Phys. 73, 357 (2001).

[36] M. H. Devoret, in Quantum Fluctuations, Les Houches Session LXIII, edited by S. Reynaud, E. Giacobino, and J. Zinn-Justin (Elsevier, New York, 1997), pp. 351-386.

[37] J. Koch, T. M. Yu, J. Gambetta, A. A. Houck, D. I. Schuster, J. Majer, A. Blais, M. H. Devoret, S. M. Girvin, and R. J. Schoelkopf, Charge-insensitive qubit design derived from the Cooper pair box, Phys. Rev. A 76, 042319 (2007).

[38] J. Bourassa, J. M. Gambetta, A. A. Abdumalikov, O. Astafiev, Y. Nakamura, and A. Blais, Ultrastrong coupling regime of cavity QED with phase-biased flux qubits, Phys. Rev. A 80, 032109 (2009).

[39] P. Nataf and C. Ciuti, Vacuum Degeneracy of a Circuit QED System in the Ultrastrong Coupling Regime, Phys. Rev. Lett. 104, 023601 (2010).

[40] T. P. Orlando, J. E. Mooij, L. Tian, C. H. van der Wal, L. S. Levitov, S. Lloyd, and J. J. Mazo, Superconducting persistentcurrent qubit, Phys. Rev. B 60, 15398 (1999).

[41] J. J. Garcia-Ripoll, B. Peropadre, and S. De Liberato, Lightmatter decoupling and A2 term detection in superconducting circuits, Sci. Rep. 5, 16055 (2015).

[42] M. Malekakhlagh and H. E. Türeci, Origin and implications of an $A^{2}$-like contribution in the quantization of circuit-QED systems, Phys. Rev. A 93, 012120 (2016).

[43] P. Macha et al., Implementation of a quantum metamaterial using superconducting qubits, Nat. Commun. 5, 5146 (2014).

[44] K. Kakuyanagi, Y. Matsuzaki, C. Deprez, H. Toida, K. Semba, H. Yamaguchi, W. J. Munro, and S. Saito, Observation of collective coupling between an engineered ensemble of macroscopic artificial atoms and a superconducting resonator, arXiv:1606.04222.

[45] T. Holstein and H. Primakoff, Field dependence of the intrinsic domain magnetization of a ferromagnet, Phys. Rev. 58, 1098 (1940).

[46] P. Forn-Diaz (private communications). 